\title{
A Modified Fuzzy C-Means Algorithm with Symmetry Information for MR Brain Image Segmentation
}

\author{
Surani Anuradha Jayasuriya and Alan Wee-Chung Liew \\ School of Information and Communication Technology, \\ Griffith University, Gold Coast Campus, \\ QLD 4222, Australia.
}

\begin{abstract}
In this paper, we present a novel modified Fuzzy Cmeans algorithm with symmetry information to reduce the effect of noise in brain tissue segmentation in magnetic resonance image (MRI). We integrate brain's bilateral symmetry into the conventional Fuzzy C-means (FCM) as an additional term. In experiments, some synthetic images, and both simulated and real brain images were used to investigate the robustness of the method against noise. Finally, the method was compared with the conventional FCM algorithm. Results show the viability of the approach and the preliminary investigation appears promising.
\end{abstract}

Keywords- Brain image segmentation; MRI; Fuzzy c-means; Brain symmetry; Mid-sagittal plane

\section{INTRODUCTION}

MRI has facilitated in vivo visualization of the brain. Many clinical diagnosis and research applications using brain MRI require a segmentation of brain images into different tissue classes. However, brain image segmentation is a complex and challenging task. Despite the number of segmentation methods in the literature $[1,2]$, the use of automated methods in clinical practice is still limited. The automated segmentation of brain tissue in MRI is a challenging task due to various image artifacts, such as noise, intensity non-uniformity (INU) and partial volume effect (PVE) [2, 3]. It is crucial to take advantage of anatomical features of the brain as a priori information for accurate segmentation.

Fuzzy c-means algorithm as a soft segmentation method is one of the most used techniques for brain image segmentation as it can handle uncertainty by incorporating fuzziness for the belongingness of each image pixel. Therefore, in comparison with crisp or hard segmentation methods, FCM can retain more information from the original image. However, conventional FCM does not consider any spatial information from the image context, which makes it sensitive to noise. Several other methods have been proposed by exploiting spatial information and modifying the standard FCM objective function $[3,4]$. These are mostly to incorporate immediate neighborhood information of a pixel. A previous work based on region-growing algorithm [5] has exploited bilateral symmetry information for image segmentation. However, they have not considered brain image segmentation. Despite the fact that the human brain exhibits approximate bilateral symmetry, to the best of our knowledge, this is the first attempt to use bilateral symmetry information for brain tissue segmentation.

Specifically, our first step is to accurately identify the brain symmetry plane $[6,7]$. The plane passing through the boundary between the two brain hemispheres is usually considered to be the mid-sagittal plane (MSP). Brain symmetry plane is generally approximated by MSP (see Fig.1). For identifying the accurate location of the MSP, we have used the method described in our previous paper [7]. In standard FCM algorithm, the classification of each pixel is determined by its intensity value. However, in our proposed method, the classification of each pixel is also influenced by the pixel in a symmetrical position with respect to the global symmetry axis. We call this pixel a mirror pixel. The aim of this paper is to show the feasibility and robustness of the algorithm by integrating the symmetry information. In experiments, some synthetic and simulated brain images were used to assess the performance of the new method in comparison with standard FCM.

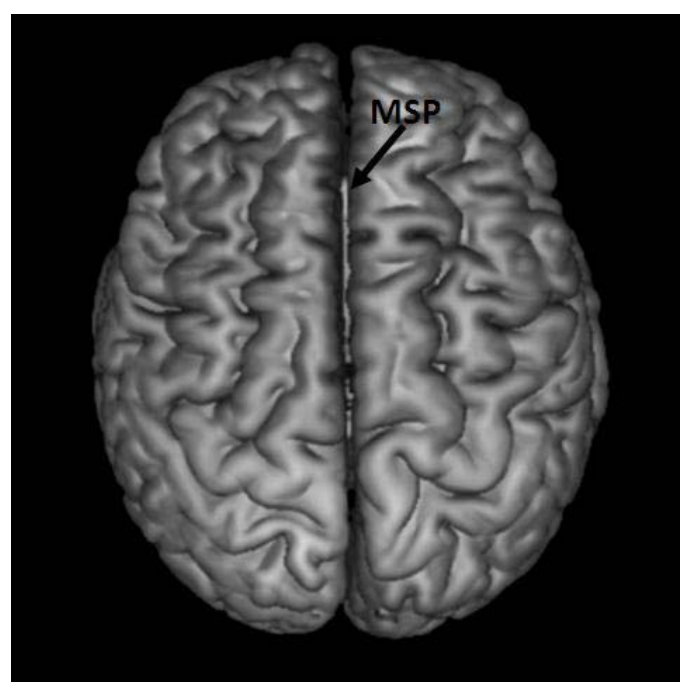

Figure 1. Brain symmetry plane/Mid-sagittal Plane (MSP): Two brain hemispheres are symmetrical about this plane. 


\section{METHODOLOGY}

\section{A. Standard Fuzzy C-means (FCM) Algorithm}

The FCM algorithm [8] assigns pixels to each category by using fuzzy memberships. Let $X=\left\{x_{k}, k=1,2, . . N\right\}$ denotes an image with $N$ pixels to be partitioned into $c$ clusters. $X_{k}$ is the intensity value of pixel $k$. The FCM algorithm is an iterative optimization that classifies $X$ into $c$ fuzzy clusters by minimizing the following objective function with respect to the membership function $\boldsymbol{u}$ and centroid values $\boldsymbol{v}$

$$
J_{F C M}=\sum_{i=1}^{c} \sum_{k=1}^{N} u_{i k}^{m}\left\|x_{k}-v_{i}\right\|^{2}
$$

$u_{i k}$ is the fuzzy membership value of pixel $k$ in cluster $i$, satisfying $0 \leq u_{i k} \leq 1$, and $\sum_{i=1}^{c} u_{i k}=1 . v_{i}(i=1, . . c)$ denotes the average intensity value in cluster $i$. $\|$.$\| is a norm metric, and$ we have considered Euclidean distance here. The parameter $m$ is a weighting exponent on each fuzzy membership and determines the amount of "fuzziness" of the resulting classification. Generally, $m=2$, and it is used in this study.

This objective function is minimized when pixels close to the centroid of their clusters are assigned high membership values while pixels far from the centroid are assigned low membership values. The update equations for minimizing $J_{F C M}$ with the necessary conditions are as follow.

$$
\begin{gathered}
u_{i k}=\frac{1}{\sum_{t=1}^{c}\left(\frac{\left\|x_{k}-v_{i}\right\|}{\left\|x_{k}-v_{t}\right\|}\right)^{2 / m-1}} \\
v_{i}=\frac{\sum_{k=1}^{N} u_{i k}^{m} x_{k}}{\sum_{k=1}^{N} u_{i k}^{m}}
\end{gathered}
$$

\section{B. Modified FCM with Symmetry Information}

The integration of information from the image context is important for accurate image segmentation. When segmenting symmetrical or nearly symmetrical image objects, this symmetry information can be utilized to give better segmentation in noisy images.

In our algorithm, we assume that the location of the global symmetry plane of the symmetrical/approximate symmetrical image is known. As mentioned previously, in brain tissue segmentation this symmetry plane is the MSP. Then, for each pixel a degree of symmetry value is computed. Although various symmetry measures can be used here [9], we defined a symmetry measure as follow $\beta_{k}=1-\frac{\lambda_{k j}}{\max (\lambda)}$

where $\lambda_{k j}=\operatorname{abs}\left(\bar{x}_{k}-\bar{x}_{j}\right)$

where $\beta_{k}$ is the degree of symmetry for $k$ th pixel, and $j$ is the mirror pixel of $k \cdot \max (\lambda)$ is the maximum value of $\lambda_{k j}$ for $k=1, . . N$. $\bar{x}_{t}$ is considered to take as median of the neighbors within a specified window around $x_{t}$.

Based on this symmetry information, our modified FCM (SymFCM) objective function can be defined as follow.

$J_{s y m F C M}=\sum_{i=1}^{c} \sum_{k=1}^{N} u_{i k}^{m}\left\|x_{k}-v_{i}\right\|^{2}+\frac{\beta_{k} u_{i k}^{m}\left\|x_{j}-v_{i}\right\|^{2}}{\alpha_{k j}}$

where

$\alpha_{k j}=1+a b s\left(x_{k}-x_{j}\right)$, and $j$ is the mirror pixel of $k$.

Thus, the update equations for minimizing $J_{\text {symFCM }}$ with the necessary conditions are as follow.

$$
u_{i k}=\frac{1}{\sum_{t=1}^{c}\left(\frac{\left(\left\|x_{k}-v_{i}\right\|^{2}+\frac{\beta_{k}\left\|x_{j}-v_{i}\right\|^{2}}{\alpha_{k j}}\right)}{\left(\left\|x_{k}-v_{t}\right\|^{2}+\frac{\beta_{k}\left\|x_{j}-v_{t}\right\|^{2}}{\alpha_{k j}}\right)}\right)^{1 / m-1}}
$$

$v_{i}=\frac{\sum_{k=1}^{N} u_{i k}^{m}\left(x_{k}+\frac{\beta_{k}}{\alpha_{k j}} x_{j}\right)}{\sum_{k=1}^{N} u_{i k}^{m}\left(1+\frac{\beta_{k}}{\alpha_{k j}}\right)}$

This modified symFCM for brain tissue segmentation can be summarized in the following steps.

Step1. Read the brain volume data.

Step2. Pre-compute the degree of symmetry matrix for the entire image.

Step3. Set the number of tissue classes $c$, select initial cluster centroids, and set the $\mathcal{E}$ to a small value $\left(1 \times 10^{-5}\right)$.

Step4. Update fuzzy membership $u_{i k}$ using eq.(6)

Step5. Update cluster centroids $v_{i}$ using eq.(7).

Step6. If $\left\|u_{i k}^{\text {new }}-u_{i k}^{\text {old }}\right\| \leq \varepsilon$, proceed to step 7 , else return to step4.

Step7. Get the final segmentation results using the maximum fuzzy membership value of each pixel.

Since the degree of symmetry can be pre-computed, the algorithm is fast and efficient. 


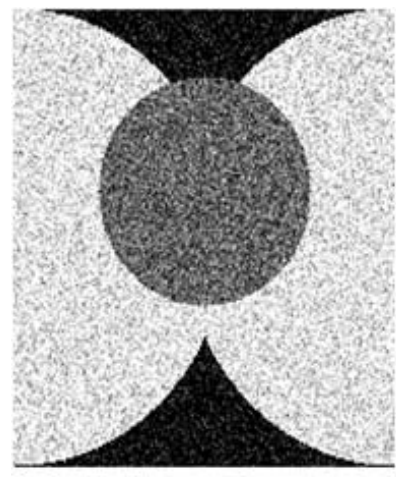

(a)

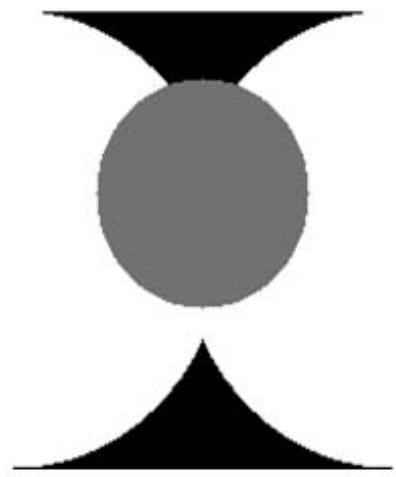

(b)

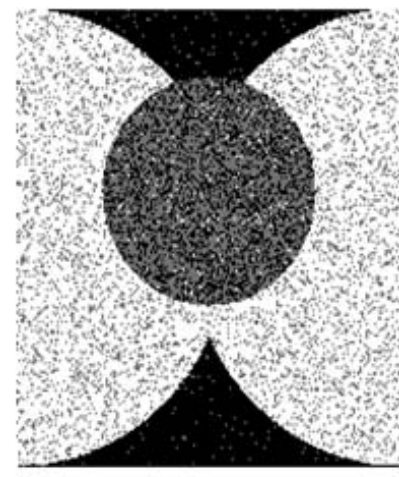

(c)

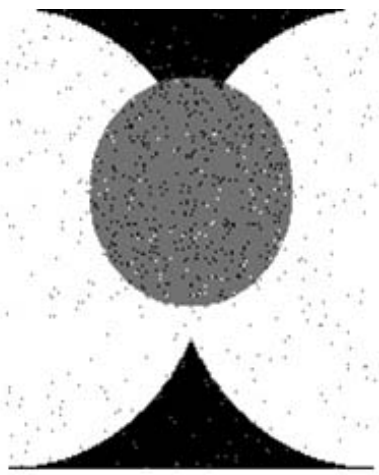

(d)

Figure 2. Comparison of segmentation results of a symmetrical synthetic grayscale image with white Gaussian noise of variance 0.03 . (a)A synthetic grayscale image with noise. (b) Ground truth. (c) Results from standard FCM. (d) Results from our method.

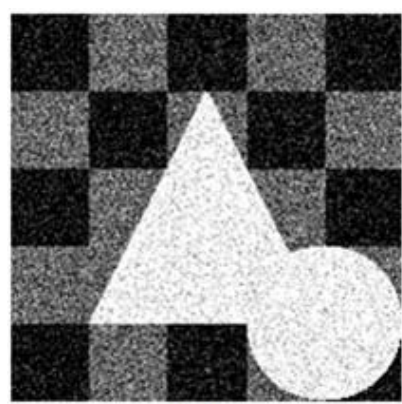

(a)

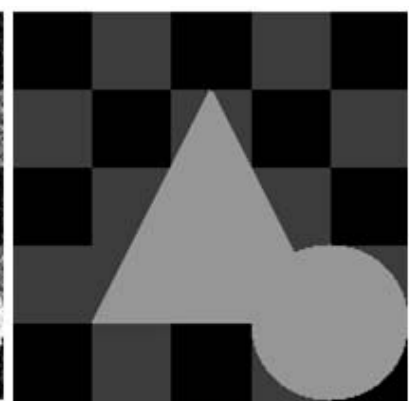

(b)

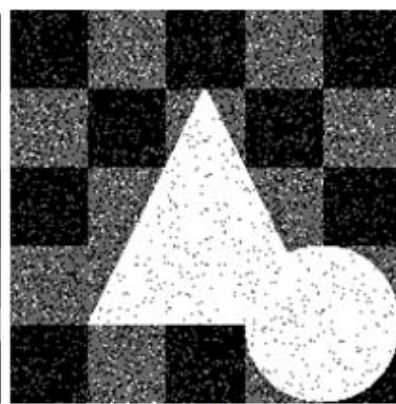

(c)

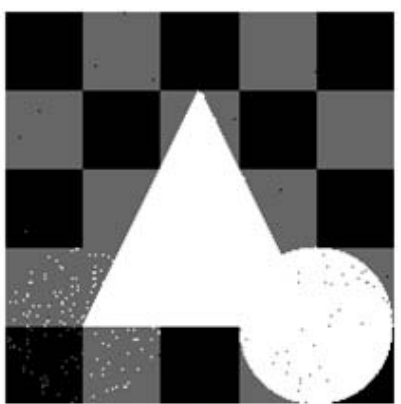

(d)

Figure 3. Comparison of segmentation results of a non-symmetrical synthetic grayscale image with white Gaussian noise with variance 0.03 . (a) A synthetic grayscale image with noise. (b) Ground truth. (c) Results from standard FCM. (d) Results from our method.

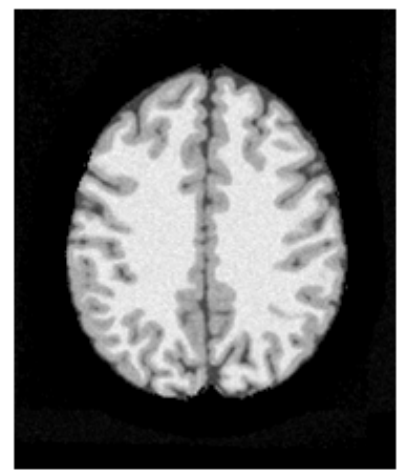

(a)

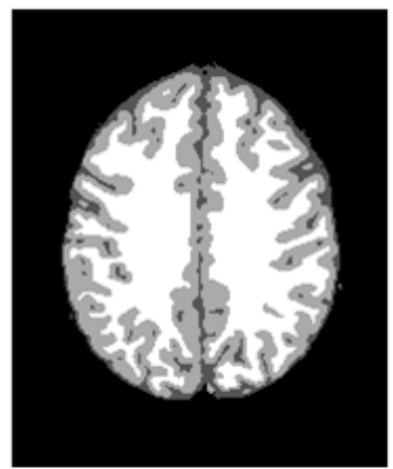

(b)

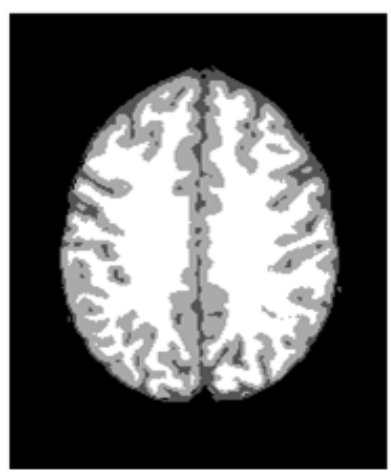

(c)

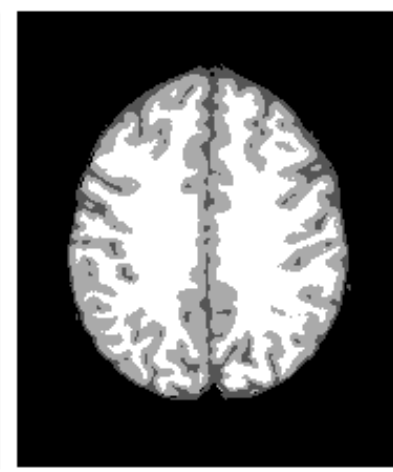

(d)

Figure 4. Comparison of segmentation results of a simulated brain MR axial slice with $3 \%$ noise, taken at $\mathrm{z}=38 \mathrm{~mm}$. (a) A simulated brain MR slice with noise $3 \%$. (b) Ground truth. (c) Results from standard FCM. (d) Results from our method. 


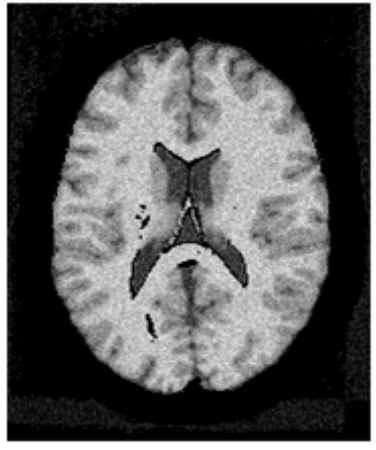

(a)

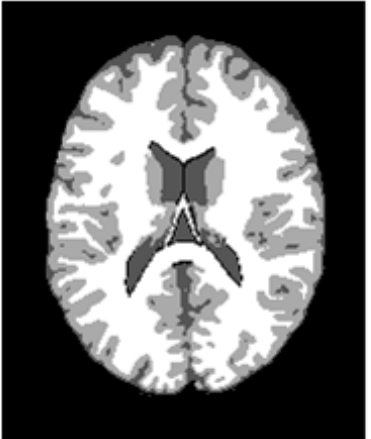

(b)

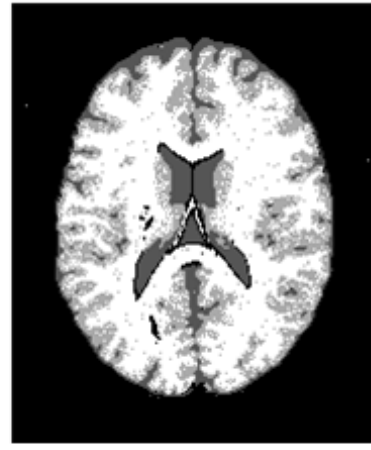

(c)

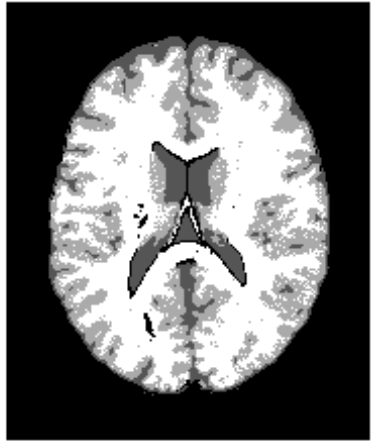

(d)

Figure 5. Comparison of segmentation results of a simulated brain MR axial slice with $9 \%$ noise, taken at z=18mm. (a) A simulated brain MR slice with noise $9 \%$. (b) Ground truth. (c) Results from standard FCM. (d) Results from our method.

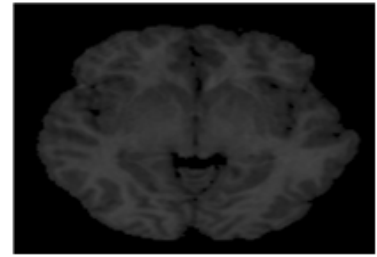

(a)

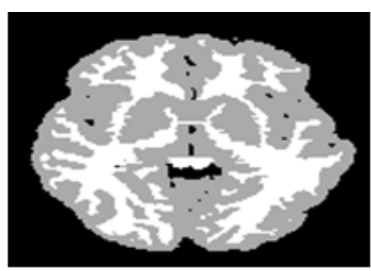

(b)

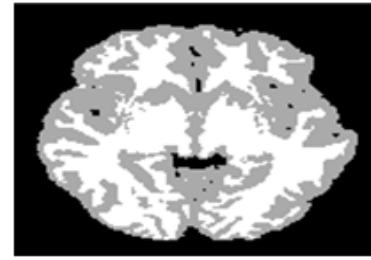

(c)

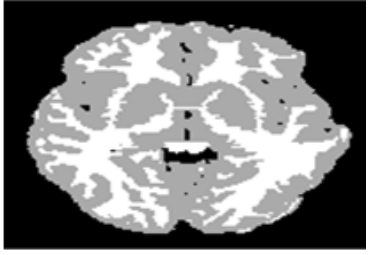

(d)

Figure 6. Comparison of segmentation results of a real T1-MR brain axial slice (taken from IBSR).

(a) Original image with INU. (b) Ground truth. (c) Results from standard FCM. (d) Results from our method.

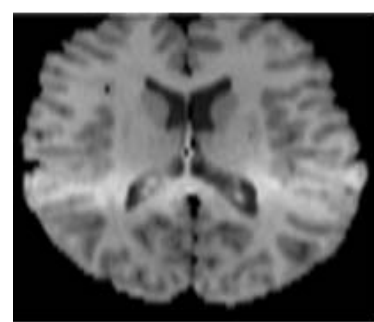

(a)

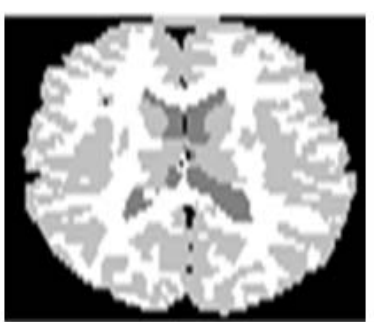

(b)

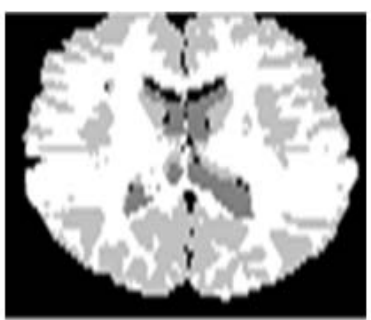

(c)

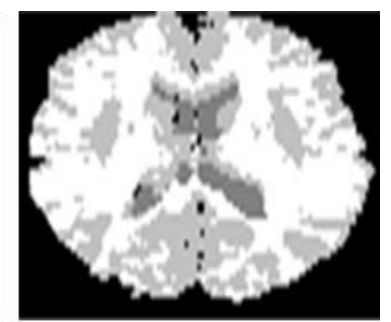

(d)

Figure 7. Comparison of segmentation results of a real T1-MR brain axial slice (taken from IBSR).

(a) Original image with INU. (b) Ground truth. (c) Results from standard FCM. (d) Results from our method.

\section{EXPERIMENTAL RESULTS}

In order to verify the validity of our approach, we used 2D synthetic grayscale images which have either a global symmetry axis or a global approximate symmetry axis. Our brain image dataset comprises of T1-weighted MR scans of normal cases. The simulated MR images were obtained from the McConell Brain Imaging Center at the Montreal Neurological Institute, McGill University [10] (http://www.bic.mni.mcgill.ca/brainweb/). The size of each image volume was $181 \times 217 \times 181($ in $\mathrm{mm}$ ) and slice thickness was $1 \mathrm{~mm}$. Phantom data with zero INU were taken from BrainWeb in order to analyze the effect of noise. Additionally, real MR brain image data were obtained from IBSR (http://www.cma.mgh.harvard.edu/) [11]. Prior to segmentation, the extra-cranial tissues such as skull and meninges were removed from all images. Then, the brain images were classified into three tissue classes: Gray Matter (GM), White Matter (WM), and Cerebrospinal Fluid (CSF).

Some of these results obtained are shown in Fig.2-Fig.7. 


\section{A. Qualitative Evaluation}

In these figures, segmentation results are compared with the segmentation obtained from standard FCM. When a qualitative comparison is done, it can be seen that the proposed method shows better segmentation in these images. When a non-symmetrical image is used as in Fig.3, although there is a misclassified region due to the consideration of symmetry, it still achieves better overall segmentation. Moreover, Fig. 6 and Fig. 7 are real images with INU.

We believe that the accuracy can further be improved when used along with spatial information, and bias field correction.

\section{B. Quantitative Evaluation}

In order to measure the quality of the segmentation performance quantitatively, we also apply segmentation accuracy measure (SA) given by,

$$
S A=\frac{\text { Number of correctly classified pixels }}{\text { Total number of pixels }} \times 100 \%
$$

Table I depicts the segmentation accuracy (SA) of the proposed method and the standard FCM when applied to synthetic images, and also simulated brain images. The SA column shows as the percentage of noise is increased, the accuracy for both methods decreases. Our method, however, consistently performs better under all noise conditions than the conventional FCM.

\section{TABLE I. SEGMENTATION ACCURACY (SA) OF EACH METHOD}

\begin{tabular}{lll}
\hline Image & FCM & symFCM \\
\hline Synthetic image1 noise variance 0.03 & $79.29 \%$ & $97.77 \%$ \\
Synthetic image2 noise variance 0.03 & $87.99 \%$ & $99.52 \%$ \\
Simulated brain image Noise 1\% & $96.28 \%$ & $96.46 \%$ \\
Simulated brain image Noise 3\% & $96.59 \%$ & $96.68 \%$ \\
Simulated brain image Noise 5\% & $92.95 \%$ & $93.10 \%$ \\
Simulated brain image Noise 9\% & $86.67 \%$ & $88.83 \%$
\end{tabular}

\section{CONCLUSION AND FUTURE WORK}

In this paper, we presented a modified fuzzy c-means algorithm that incorporates symmetry information in order to improve the segmentation results. Degree of symmetry was computed for each pixel/voxel about the global symmetry axis/plane. This information was transformed into a weighting function which was incorporated into the objective function.
The new method was tested on synthetic images as well as simulated and real MR brain images. Preliminary results showed that the effect of noise in segmentation was considerably less with the new algorithm than with the conventional FCM.

For future work, we would incorporate spatial information into the current objective function and take into account the intensity non-uniformity to further improve the segmentation accuracy.

\section{ACKNOWLEDGMENT}

This work was supported by the Australian Research Council (ARC) Discovery Grant DP1097059 and the Australian Postgraduate Award (APA).

\section{REFERENCES}

[1] J. Bezdek, L. Hall, and L. Clarke. "Review of MR Image Segmentation Techniques using Pattern Recognition," Medical Physics, 20: pp. 1033$1048,1993$.

[2] A. W. C. Liew and H. Yan. "Current Methods in the Automatic Tissue Segmentation of 3D Magnetic Resonance Brain Images," Current Medical Imaging Reviews, 2(1): pp. 91-103, 2006.

[3] A.W. C. Liew and H. Yan. "An Adaptive Spatial Fuzzy Clustering Algorithm for MR Image Segmentation," IEEE Transactions on Medical Imaging, 22(9): pp. 1063-1075, 2003.

[4] M. Ahmed, S. Yamany, N. Mohamed, A. Farag, and T. Moriarty. "A Modified Fuzzy C-Means Algorithm for Biasfield Estimation and Segmentation of MRI Data," IEEE Transactions on Medical Imaging, 21(3): pp. 193-199, 2002.

[5] Y. Sun and B. Bhanu. "Reflection Symmetry-integrated Image Segmentation," IEEE Transactions on Pattern Analysis and Machine Intelligence, 34(9): pp. 1827-1841, 2012.

[6] S. A.Jayasuriya and A. W. C. Liew. "Symmetry Plane Detection in Neuroimages based on Intensity Profile Analysis," In IEEE International Symposium on Information Technology in Medicine and Education (ITME), volume 2, pp. 599-603, 2012.

[7] S. A.Jayasuriya and A. W. C. Liew. "Fractal Dimension as a Symmetry Measure in 3D Brain MRI Analysis," In IEEE International Conference on Machine Learning and Cybernetics, pp. 1118-1123, 2012.

[8] J. Bezdek. Pattern Recognition with Fuzzy Objective Function Algorithms. Plenum Press, 1981.

[9] D. O’Mara and R. Owens. "Measuring Bilateral Symmetry in Digital Images," In IEEE TENKON- Digital Signal Processing Applications, pp. 151-156, 1996

[10] C. Cocosco, V. Kollokian, R. Kwan, and A. Evans. "Brainweb: Online Interface to a 3D MRI Simulated Brain Database," Neuroimage, 5(4): pp. 425-425, 1997.

[11] IBSR; Available from: http://www.cma.mgh.harvard.edu/ibsr/ 